\title{
Fusion between cancer cells and myofibroblasts is involved in osteosarcoma
}

\author{
LING YU, WEICHUN GUO, SHENGHAO ZHAO, FUAN WANG and YONG XU \\ Department of Orthopedics, Renmin Hospital of Wuhan University, Wuhan, Hubei, P.R. China
}

Received February 22, 2011; Accepted July 5, 2011

DOI: $10.3892 / \mathrm{ol} .2011 .363$

\begin{abstract}
Communication between cancer cells and the microenvironment appears to be an important determinant of disease prognosis. However, the detailed mechanisms of the interactions between cancer cells and surrounding cells have yet to be clarified. Recent studies on cell fusion have indicated this interaction to be one of the driving forces in cancer progression. Fibroblasts constitute a significant component of the carcinoma stromal compartment. Many of these fibroblasts are thought to differentiate into myofibroblasts, which are characterized by a positive expression of $\alpha$-smooth muscle actin. Expression of $\alpha$-smooth muscle actin in osteosarcoma was evaluated, and was observed to be excessive in the multinucleated osteoclast-like giant cells in osteosarcoma tissue, indicating the possibility of cell fusion between cancer cells and myofibroblasts. In order to test the above hypothesis, we first transformed the primary mouse embryonic fibroblast cells into activated myofibroblast cells. Osteosarcoma cells were then co-cultured with mouse myofibroblast cells, and cell fusion was investigated using species-specific chromosomal markers. Expression of $\alpha$-smooth muscle actin was successfully induced in primary mouse embryonic fibroblast cells. Cells fused spontaneously with a fusion rate of approximately $1-2 \%$ and fusion between more than two cells was also observed. Our study demonstrated that fusion between cancer cells and myofibroblasts may contribute to the observed multinucleated giant cells in osteosarcoma. We posit that cell fusion is a novel mechanism for the interaction between cancer cells and the microenvironment.
\end{abstract}

\section{Introduction}

Osteosarcoma originates from primitive bone-forming mesenchymal cells and is the most prevalent primary bone malignancy. It ranks eighth in general incidence among childhood cancers (1). The overall 5-year survival rate for

Correspondence to: Professor Weichun Guo, Department of Orthopedics, Renmin Hospital of Wuhan University, 99 Ziyang Road, Wuhan, Hubei, P.R. China

E-mail: guoweichun@yahoo.com.cn

Key words: osteosarcoma, cell fusion, $\alpha$-smooth muscle actin, myofibroblast osteosarcoma is $68 \%$. Certain genetic predispositions have been observed to correlate with osteosarcoma, including hereditary retinoblastoma and Li-Fraumeni syndrome, which are characterized by a high risk of developing osteosarcoma $(2,3)$. Genetic aberrations that accompany osteosarcoma have been identified; however, osteosarcoma is characterized by karyotypes which exhibit a high degree of complexity $(4,5)$. Understanding these mechanisms is clearly crucial to osteosarcoma therapy.

Cell fusion plays a crucial role in homeostasis, such as fertilization, formation of placenta, bone and muscle tissues and tissue repair and regeneration (6). It was first thought to be involved in tumorigenesis by Otto Aichel in the early 1900s, who posited that fusion between somatic cells may result in chromosomal abnormalities in cancer. Recent discoveries of cell fusion in tissue homeostasis and regeneration have revitalized the interest in cell fusion as one of the driving forces of cancer progression (7). Stroma surrounding cancer cells plays a supportive role in tumor development and progression. The osteosarcoma stroma histopathologically comprises various supportive components, including fibro-blasts, inflammatory cells, immune cells, smooth muscle cells and endothelial cells. Fibroblasts form a significant component of the stromal compartment. A number of these fibroblasts are differentiated into carcinoma-associated fibroblasts (CAF), as with so-called 'myofibroblasts'. Myofibroblasts are thought to contain both fibroblast and smooth muscle cell characteristics, which are defined by positive expression of both stromal cell type markers and smooth muscle cell markers (8). $\alpha$-smooth muscle actin is one of the markers that is widely used to detect myofibroblast.

When investigating the expression of $\alpha$-smooth muscle actin in osteosarcoma tissues, we observed that it was excessively expressed in multinucleated cells in osteosarcoma tissue. This expression indicates the involvement of cancer cell fusion with myofibroblast. However, little evidence is currently available that is of any relevance to osteosarcoma. This is therefore the first study to demonstrate that human osteosarcoma cells are capable of fusing with myofibroblast cells to form hybrid cells in vitro.

\section{Materials and methods}

Patients and specimens. Informed consent was obtained from the patients or their relatives, as appropriate. This investigation 
was performed according to the guidelines approved by the Institutional Animal Care and Use Committee. A total of 12 paraffin wax-embedded specimens from patients with osteosarcoma were collected from the Renmin Hospital of Wuhan University, Wuhan, Hubei, China, between January 2007 and June 2009. All cases were confirmed by pathological diagnosis.

Immunohistochemistry. Sections were deparaffinized and rehydrated using graded alcohols. Antigen retrieval was performed by boiling the slides in $10 \mathrm{mM}$ citrate buffer (pH 6.0) for $10 \mathrm{~min}$. Cells seeded on the slides were fixed in $4 \%$ paraformaldehyde in phosphate-buffered saline (PBS) and permeabilized in $0.1 \%$ Triton X-100. Endogenous peroxidase activity was blocked with $3 \%$ hydrogen peroxide. The slides or cells were then incubated in a humid chamber with the rabbit anti-human/mouse $\alpha$-smooth muscle actin polyclonal antibody (ProteinTech Group, Chicago, IL, USA) at a dilution of 1:100, at $4^{\circ} \mathrm{C}$, overnight. The slides were then incubated for $30 \mathrm{~min}$ at $37^{\circ} \mathrm{C}$ with horseradish peroxidase-labeled goat anti-rabbit antibody. The slides were developed using DAB, and then counterstained with haematoxylin, dehydrated through graded alcohols, air-dried and mounted in neutral resins. Some slides were stained by AEC, then directly mounted using aqueous mounting solution. Primary antibodies were substituted with PBS in the negative controls.

Cell culture. The human osteosarcoma cell lines U2OS and MG63 were purchased from the Shanghai Institute for Biological Sciences of Chinese Academy of Sciences. They were cultured in Dulbecco's modified Eagle's medium (DMEM; high glucose) supplemented with $10 \%$ fetal bovine serum. The cells were cultured in a $37^{\circ} \mathrm{C}$ humidified incubator with a mixture of $95 \%$ air and $5 \% \mathrm{CO}_{2}$. Primary mouse embryonic fibroblasts (PMEFs) were established and maintained according to protocol by Garfield (9). In brief, the mice were sacrificed on postcoitum day E14.5. Each embryo was removed and placed in prewarmed complete medium, decapitated and eviscerated, and as much blood and liver tissue was discarded as possible. Trypsin/EDTA was added to the remainder after homogenization. Supernatant was collected following adequate precipitation, and was centrifuged at $1000 \mathrm{rpm}$ for 10 min to obtain the cell pellet. The pellet was resuspended and $1 \times 10^{6}$ cells were seeded into a $25-\mathrm{cm}^{2}$ flask. Cell maintenance and splitting were manipulated routinely. For induction of myofibroblasts, the cells were harvested and washed with $0.1 \%$ BSA serum-free medium. U2OS or MG63 cells were seeded on the upper chamber with complete medium as an inducer. The PMEFs were placed into the bottom chamber of a transwell unit (Corning Costar, Corning, NY, USA). The polycarbonate $3 \mathrm{~mm}$ pore membrane was precoated with $0.2 \mathrm{mg} / \mathrm{ml}$ of rat tail type I collagen (BD Biosciences, Bedford, MA, USA) to inhibit the migration of cancer cells. The cells were allowed to culture at $37^{\circ} \mathrm{C}$ and $5 \% \mathrm{CO}_{2}$ for 28 days and the medium was changed twice a week. For co-cultures of both osteosarcoma cells and myofibroblasts, cells were harvested by trypsinization and equal numbers of cancer and myofibroblast cells were mixed. Cells were grown on sterile glass slides (Nalge Nunc, Naperville, IL, USA), fixed in $4 \%$ paraformaldehyde in PBS and permeabilized in $0.1 \%$ Triton
$\mathrm{X}-100$ for immunohistochemistry or in methanol-acetic acid for fluorescence in situ hybridization (FISH).

Western blot analysis. Cells were washed with ice-cold PBS after trypsinization and centrifuged at $1000 \mathrm{rpm}$ for $10 \mathrm{~min}$ at room temperature. The pellet containing approximately $1 \times 10^{6}$ cells was lysed in $100 \mu$ l of RIPA cell lysis buffer containing protease inhibitors and quantified by the BCA method. Protein $(100 \mu \mathrm{g})$ was separated by SDS-PAGE and transferred to nitrocellulose membranes. After blocking with 5\% skim milk, the membranes were incubated with the following primary antibodies: polyclonal rabbit anti- $\alpha$-smooth muscle actin (1:1000; Santa Cruz Biotechnology, Santa Cruz, CA, USA) and monoclonal mouse anti- $\beta$-actin (1:2500; Sigma, St. Louis, MO, USA). The blots were then incubated with horseradish peroxidase-conjugated secondary antibody. Enhanced chemiluminescence was used for detection and developed by X-ray film.

Fluorescent in situ hybridization. The cells were fixed in methanol-acetic acid and predenatured, dehydrated, denatured, and hybridized with DNA probes for mouse 8 and human $\mathrm{X}$ chromosomes (ID Labs, London, Ontario, Canada) overnight at $37^{\circ} \mathrm{C}$ in a humidified chamber. After a post-hybridization wash, slides were counterstained with 4,6-diamidino-2-phenylindole and examined using an epifluorescence microscope (Nikon Eclipse TE2000-U).

Statistical analysis. The Student's t-test was used to compare the means of the 2 groups. When $\geq 3$ means were compared, one-way ANOVA followed by multiple comparisons among the means was used.

\section{Results}

$\alpha$-smooth muscle actin expression in osteosarcoma specimens. Table I shows the patient characteristics. To assess the state of $\alpha$-smooth muscle actin expression in clinical osteosarcoma samples, immunohistochemical staining was performed. Marked $\alpha$-smooth muscle actin expression was observed in specimens from osteosarcoma patients compared with normal bone tissue. Marked staining was observed in $90 \%(9 / 10)$ of the primary osteosarcoma. Slight staining was detected in $30 \%(3 / 10)$ of normal bone tissue. Most notably, a distinctive staining pattern was observed depending on the nuclei status of the cells. Numerous multinucleated cells were observed in the osteosarcoma tissue (4/10), 3 of which were from histologically low-differentiated osteosarcoma patients. These cells were markedly cytoplasmic-positive for $\alpha$-smooth muscle actin (Fig. 1).

$\alpha$-smooth muscle actin expression in osteosarcoma cell lines. Expression of $\alpha$-smooth muscle actin was examined in osteosarcoma cell lines MG63 and U2OS to confirm the above result. $\alpha$-smooth muscle actin was found to be widely expressed in the osteosarcoma cell line, was likely to form particles, and was mainly observed in cytoplasm (Fig. 1).

Osteosarcoma cells activated the PMEFs. We investigated the expression of $\alpha$-smooth muscle actin in PMEFs and noted that 
Table I. Patient characteristics.

\begin{tabular}{lr}
\hline Total & 10 \\
Gender & \\
Male & 6 \\
Female & 4 \\
Age & \\
Mean & 28.6 \\
Range & $9-57$ \\
Location & \\
Humerus & 5 \\
Femur & 2 \\
Scapula & 1 \\
Skull & 1 \\
Iliac & 1 \\
Pathological variables & \\
Types of tumor & \\
Osteoblastic & 5 \\
Chondroblastic & \\
Fibroblastic & 2 \\
Histological grade & 3 \\
Well & \\
Moderate & \\
Poor & 2 \\
\hline
\end{tabular}

there was no staining in PMEFs using immunohistochemistry and Western blotting methods. To observe whether osteosarcoma was capable of transforming PMEFs to myofibroblasts, U2OS or MG63 cells were co-cultured with PMEFs using a transwell unit. Expression of $\alpha$-smooth muscle actin in PMEFs was detected 28 days after placing into the bottom chamber by both immunohistochemistry and Western blotting, and vimentin was also induced in the co-culture groups. Both $\alpha$-smooth muscle actin and vimentin were found to be expressed in cytoplasm (Fig. 2).

Spontaneous fusion between osteosarcoma and myofibroblast cells in vitro. Equal numbers of human osteosarcoma cancer (U2OS and MG63) cells and PMEFs were co-cultured. FISH was performed using point FISH probes specific to human chromosome $\mathrm{X}(\mathrm{hCX})$ and mouse probes specific to mouse chromosome 8 ( $\mathrm{mC} 8)$, labeled with fluorochrome delivering green and red fluorescence, respectively. This process allowed for the detection of $\mathrm{hCX}$ and $\mathrm{mC} 8$ in contrasting colors. Double fluorescence for species-specific chromosomal markers revealed that $\mathrm{mC} 8$ did not occur in U2OS cultured alone, and hCX did not appear in mouse myofibroblast cells cultured alone. However, in co-cultures, $3.0 \pm 2.3 \%$ of all hCXpositive cells were also $\mathrm{mC} 8$-positive and $2.9 \pm 2.3 \%$ of all mC8-positive cells were also hCX-positive, with a total fusion rate of $1.4 \pm 0.98 \%$. Co-cultures of MG63 cells and mouse myofibroblast cells revealed a slightly higher total fusion rate of $1.8 \pm 1.5 \%$. Mouse myofibroblast cells consistently had two copies of mC8. Due to different gender origins, the U2OS cells each possessed two copies of hCX, while MG63 cells had one. When MG63 cells and mouse myofibroblast cells

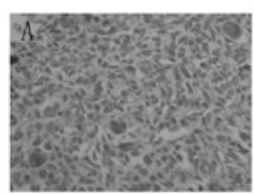

DAB stain

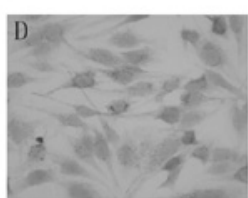

U2OS

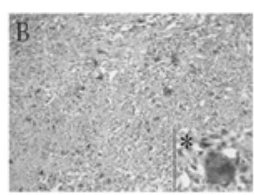

AEC stain

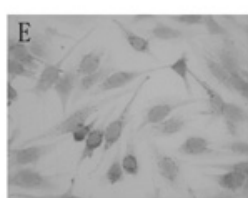

MG63

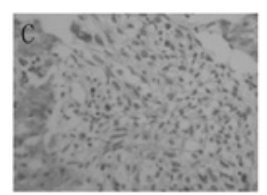

$\mathrm{NC}$

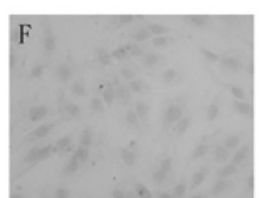

NC
Figure 1. $\alpha$-smooth muscle actin ( $\alpha$-sma) expression in osteosarcoma. (A-C) Representative samples of the staining for $\alpha$-sma developed by DAB and AEC in osteosarcoma, with the corresponding negative control (magnification, $\mathrm{x} 400$ ). (D-F) Staining for $\alpha$-sma in U2OS and MG63 cells, and the negative control (magnification, $x 400$ ). *Magnification, x200; with $x 400$ on the low right inset.

A $\alpha$-sma

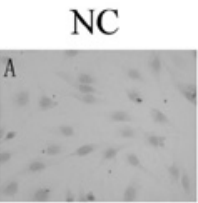
$+\mathrm{U} 2 \mathrm{OS}$

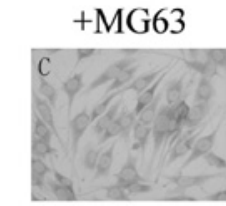

vimentin
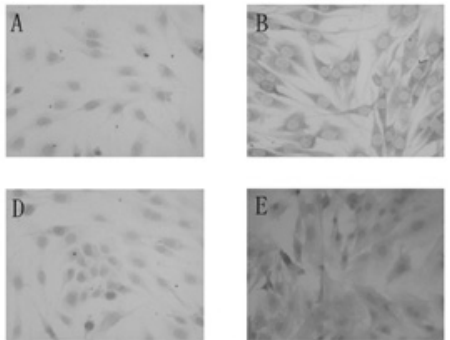

B
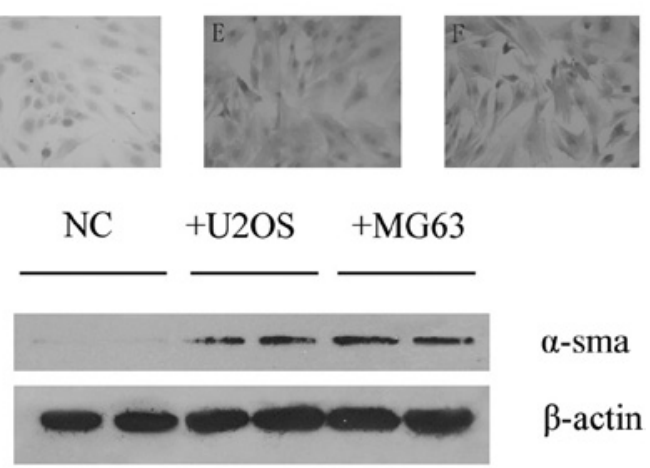

Figure 2. PMEFs were activated by osteosarcoma cell lines. (A) Immunohistochemistry of $\alpha$-sma and vimentin in PMEFs following exposure to U2OS and MG63 cells. Complete medium without cancer cells was added as a negative control. (B) Detection of $\alpha$-sma expression in PMEFs using Western blotting. Complete medium without cancer cells was added as a negative control.

were co-cultured, it was observed that certain cells contained two copies of $\mathrm{hCX}$ and $\mathrm{mC}$, indicating fusion between two MG63 cells and one mouse myofibroblast cell. These data revealed that cancer and myofibroblast cells are able to fuse to form hybrid cells (Fig. 3).

\section{Discussion}

High-grade conventional osteosarcoma cells are marked by nuclear pleiomorphism, conspicious chromatin abnormalities and prominent nucleoli. (10). In our study, osteosarcoma also presented multinucleated osteoclast-like giant cells, which are more likely to exhibit low differentiation. However, the origin and mechanisms of multinucleated giant cells in osteosarcoma are poorly understood. Spontaneous cell fusion 
A

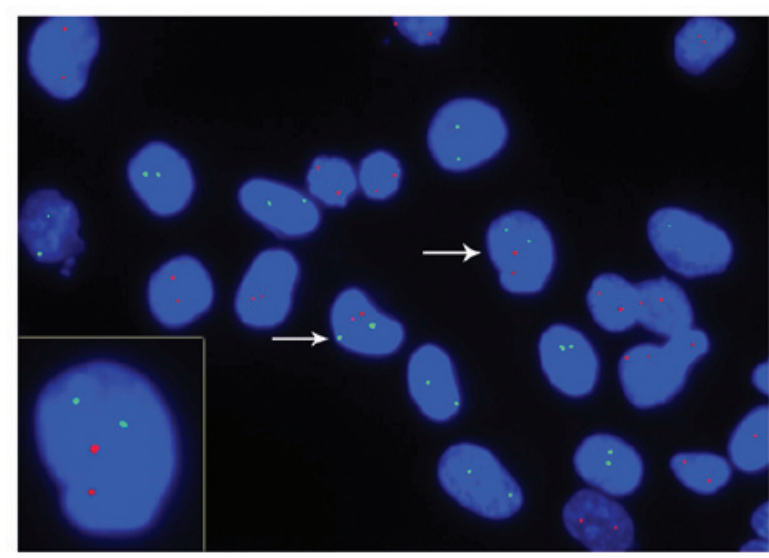

U2OS+tPMEF
B

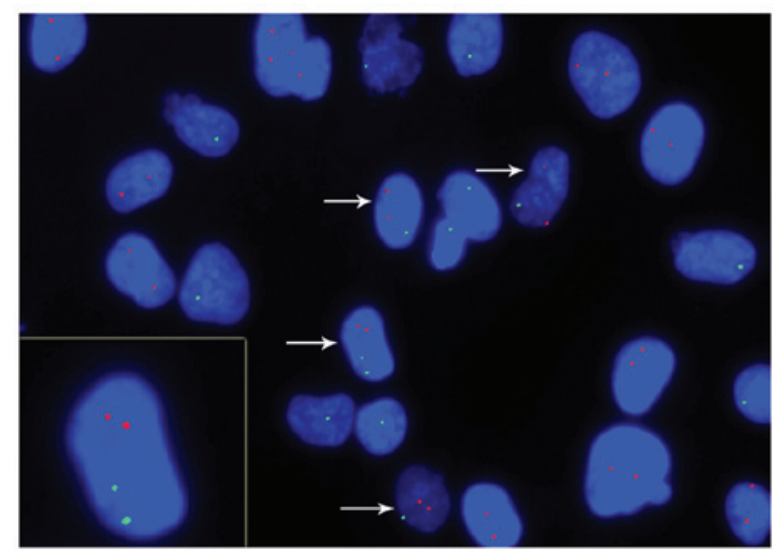

MG63+tPMEF

Figure 3. Co-cultures of (A) U2OSor (B) MG63 cells with mouse myofibroblast cells were submitted to double FISH detecting human chromosome X (green) and mouse chromosome 8 (red). DNA is visualized by DAPI staining (blue). Co-culture of U2OS cells with mouse myofibroblast cells show a pair of fused cells containing two copies of hCX and mC8. (A) Additionally, non-fused U2OS cells were observed, which contain two copies of hCX, and non-fused PMEF cells, which contain two copies of mC8. (B) Co-culture of MG63 cells with mouse myofibroblast cells shows four fused cells contained both hCX and mC8. Non-fused MG63 cells contained only one copy of hCX and non-fused mouse myofibroblast cells contained two copies of mC8. One fused cell containing two copies of hCX is shown.

in tissue culture or in animal models has been reported in a wide variety of tumor cells. Similar to the formation of multinuclear osteoclasts for bone resorption, cell fusion is likely to be the origin of the multinucleated osteoclast-like giant cells in osteosarcoma. $\alpha$-smooth muscle actin expression was investigated in osteosarcoma and was found to be markedly expressed in multinucleated osteoclast-like giant cells in osteosarcoma specimens. $\alpha$-smooth muscle actin is commonly used as a marker of myofibroblasts. We therefore speculate that the giant cells were the result of fusion between osteosarcoma cancer cells and myofibroblasts.

The tumor microenvironment contains multiple types of cells, among which myofibroblasts are attracting increasing attention. Communications between cancer cells and the microenvironment appears to be a significant determinant of disease outcome. In colorectal cancer, both $\alpha$-smooth muscle actin and FAP expression are associated with poor prognosis, and the two proteins are expressed in CAF or myofibroblasts $(11,12)$. Similarly, elevated FAP or SPARC expression correlates with poor outcome in patients with pancreatic cancer $(13,14)$. Myofibroblasts play a crucial role in carcinogenesis as recipients and producers of pro-tumorigenic signals $(15,16)$. Myofibroblasts form the source of many well-known tumorpromoting factors, including EGF, TGF $\beta$ or HGF. Previous studies have demonstrated that myofibroblasts affect sensitivity of malignant cells to chemo- or radiotherapy $(17,18)$ and have a direct pro-metastatic effect (19).

Since $\alpha$-smooth muscle was found to express in the osteosarcoma cell lines U2OS and MG63, largely due to its mesenchymal origin, it is difficult to identify in cell cultures using lineage-specific tracking markers. Although previous studies have demonstrated that myofibroblasts are derived from bone marrow-derived (20,21), epithelial (22) or endothelial cells (23), local fibroblasts or fibroblast precursors have been considered to be the major source of myofibroblasts. Activated primary mouse embryonic fibroblasts were therefore co-cultured with osteosarcoma cells and the fusion rate was investigated. This fusion was examined using probes tagging different chromosomes according to their different genus. Mouse myofibroblasts were successfully induced by culturing osteosarcoma and PMEFs in separate chambers of a transwell unit. Increased expression of $\alpha$-smooth muscle actin and vimentin in PMEFs was noted 28 days post-induction, while the naïve or negative control exhibited little or no expression. Our data are in agreement with those of Mishra et al (24), who exposed bone marrow-derived mesenchymal stem cells to tumor-conditioned medium and succeeded in transforming the cells into activated CAF.

Experimental and clinical studies suggest a potentially multifaceted involvement of cell fusion in tumor initiation and progression. Spontaneous cell fusion in vitro or in vivo has been reported in a variety of tumors. The frequency of cell fusion can be up to $1 \%$ in vivo in experimental tumor models. Furthermore, fusion efficiency is proportional to the malignant level of tumor cells $(25,26)$. Andersen et al (27) examined the karyotype of renal-cell carcinoma patients who received allogeneic bone-marrow transplantation from the opposite gender. The results showed that they had adopted chromosomes from the bone marrow donors. This is the most definitive and direct evidence for involvement of cell fusion in human cancer. Despite the rarity of direct evidence of cell fusion in human cancer, increasing experimental evidence has indicated a broad involvement of cell fusion. In non-programmed accidental fusion, the two nuclei may fuse and lead to aneuploidy and potentially cancer, given the existence of other genetic alterations. Binuclear and multinuclear cells are frequently observed in many types of tumors and cell fusion is likely to be one of several mechanisms generating 
such cells. In the present study, equal numbers of osteosarcoma and mouse myofibroblast cells were mixed and plated in the same flask. The fusion rate was investigated using FISH 2 days after co-culture. We provide definite evidence that human ostesosarcoma cells fuse with myofibroblast cells and result in hybrid cells that contain chromosome markers characterizing both fusion partners. Mortensen et al (28) co-cultured breast cancer and endothelial cells and confirmed the existence of spontaneous fusion between cancer cells and cells that may have originated in the microenvironment. Their results are supported by our data, which indicate the potential significance of cell fusion in tumor development and progression.

Taken together, we determined that fusion between cancer cells and myofibroblasts may contribute to observed multinucleated giant cells in osteosarcoma and we propose that cell fusion is a novel mechanism for the interaction between cancer cells and the microenvironment.

\section{References}

1. Ottaviani $\mathrm{G}$ and Jaffe $\mathrm{N}$ : The epidemiology of osteosarcoma Cancer Treat Res 152: 3-13, 2009.

2. Hansen MF, Koufos A, Gallie BL, et al: Osteosarcoma and retinoblastoma: a shared chromosomal mechanism revealing recessive predisposition. Proc Natl Acad Sci USA 82: 6216-6120, 1985.

3. Porter DE, Holden ST, Steel CM, Cohen BB, Wallace MR and Reid R: A significant proportion of patients with osteosarcoma may belong to Li-Fraumeni cancer families. J Bone Joint Surg Br 74: 883-886, 1992.

4. Selvarajah S, Yoshimoto M, Ludkovski O, et al: Genomic signatures of chromosomal instability and osteosarcoma progression detected by high resolution array CGH and interphase FISH. Cytogenet Genome Res 122: 5-15, 2008.

5. Bayani J, Zielenska M, Pandita A, et al: Spectral karyotyping identifies recurrent complex rearrangements of chromosomes 8 17, and 20 in osteosarcomas. Genes Chromosomes Cancer 36: 7-16, 2003.

6. Ogle BM, Cascalho M and Platt JL: Biological implications of cell fusion. Nat Rev Mol Cell Biol 6: 567-575, 2005.

7. Lu X and Kang Y: Cell fusion as a hidden force in tumor progression. Cancer Res 69: 8536-8539, 2009.

8. Orimo A, Tomioka Y, Shimizu Y, et al: Cancer-associated myofibroblasts possess various factors to promote endometrial tumor progression. Clin Cancer Res 7: 3097-3105, 2001.

9. Garfield AS: Derivation of primary mouse embryonic fibroblast (PMEF) cultures. Methods Mol Biol 633: 19-27, 2010

10. Meister P, Konrad E, Lob G, Janka G, Keyl W and Stürz H: Osteosarcoma: histological evaluation and grading. Arch Orthop Trauma Surg 94: 91-98, 1979.
11. Tsujino T, Seshimo I, Yamamoto H, et al: Stromal myofibroblasts predict disease recurrence for colorectal cancer. Clin Cancer Res 13: 2082-2090, 2007.

12. Henry LR, Lee HO, Lee JS, et al: Clinical implications of fibroblast activation protein in patients with colon cancer. Clin Cancer Res 13: 1736-1741, 2007.

13. Cohen SJ, Alpaugh RK, Palazzo I, et al: Fibroblast activation protein and its relationship to clinical outcome in pancreatic adenocarcinoma. Pancreas 37: 154-158, 2008.

14. Infante JR, Matsubayashi H, Sato N, et al: Peritumoral fibroblast SPARC expression and patient outcome with resectable pancreatic adenocarcinoma. J Clin Oncol 25: 319-325, 2007.

15. Sasaki T, Nakamura T, Rebhun RB, et al: Modification of the primary tumor microenvironment by transforming grow th factor alpha-epidermal growth factor receptor signaling promotes metastasis in an orthotopic colon cancer model. Am J Pathol 173: 205-16, 2008.

16. Kalluri R and Zeisberg M: Fibroblasts in cancer. Nat Rev Cancer 6: 392-401, 2006.

17. Shekhar MP, Santner S, Carolin KA and Tait L: Direct involvement of breast tumor fibroblasts in the modulation of tamoxifen sensitivity. Am J Pathol 170: 1546-1560, 2007.

18. Koukourakis MI, Giatromanolaki A, Harris AL and Sivridis E: Comparison of metabolic pathways between cancer cells and stromal cells in colorectal carcinomas: a metabolic survival role for tumor-associated stroma. Cancer Res 66: 632-637, 2006.

19. Karnoub AE, Dash AB, Vo AP, et al: Mesenchymal stem cells within tumour stroma promote breast cancer metastasis. Nature 449: 557-563, 2007.

20. Ishii G, Sangai T, Oda T, et al: Bone-marrow-derived myofibroblasts contribute to the cancer-induced stromal reaction. Biochem Biophys Res Commun 309: 232-240, 2003.

21. Direkze NC, Hodivala-Dilke K, Jeffery R, et al: Bone marrow contribution to tumor-associated myofibroblasts and fibroblasts. Cancer Res 64: 8492-8495, 2004.

22. Radisky DC, Kenny PA and Bissell MJ: Fibrosis and cancer: do myofibroblasts come also from epithelial cells via EMT? J Cell Biochem 101: 830-839, 2007.

23. Zeisberg EM, Potenta S, Xie L, Zeisberg M and Kalluri R: Discovery of endothelial to mesenchymal transition as a source for carcinoma-associated fibroblasts. Cancer Res 67: 10123-10128, 2007.

24. Mishra PJ, Mishra PJ, Humeniuk R, et al: Carcinoma-associated fibroblast-like differentiation of human mesenchymal stem cells. Cancer Res 68: 4331-4339, 2008.

25. Miller FR, McInerney D, Rogers C and Miller BE: Spontaneous fusion between metastatic mammary tumor subpopulations. J Cell Biochem 36: 129-136, 1988.

26. Duelli D and Lazebnik Y: Cell fusion: a hidden enemy? Cancer Cell 3: 445-448, 2003.

27. Andersen TL, Boissy P, Sondergaard TE, et al: Osteoclast nuclei of myeloma patients show chromosome translocations specific for the myeloma cell clone: a new type of cancer-host partnership? J Pathol 211: 10-17, 2007.

28. Mortensen K, Lichtenberg J, Thomsen PD and Larsson LI: Spontaneous fusion between cancer cells and endothelial cells. Cell Mol Life Sci 61: 2125-2131, 2004. 